\title{
Stabilization of Biped Dynamic Walking Using Gyroscopic Couple
}

\author{
Terence C. F. Wong and Y. S. Hung \\ Department of Electrical and Electronic Engineering \\ The University of Hong Kong \\ Hong Kong \\ email: twong@hkueee.hku.hk, yshung@hkueee.hku.hk
}

\begin{abstract}
This paper presents a new strategy for dynamic walking where a gyroscope mounted on top of the biped is precessed to achieve balancing both in the Coronal and Sagittal planes. A gait is devised and simulation results are provided to show the feasibility of proposed balancing method.
\end{abstract}

Keywords: biped, dynamic walking, gyroscope

\section{Introduction}

Gyroscope is one of the most common sensors used for navigation, e.g., in aircraft and ships. It has also been used as an attitude sensor on walking robots[1]. Another kind of application of the gyroscope is for motion stabilization, e.g., in monorail cars and ships[4].

Much research on locomotion of bipeds (two-legged walking robots) has been conducted in the past years. Both static and dynamic walking methods have been developed. The balance of bipeds in the Coronal (frontal) plane, often neglected in simulations of 2D walking, is of great importance. The majority of the proposed solutions to the balancing problem in the Coronal plane can be categorized into two groups. The first group is to use physical means to restrict the movement in the Coronal plane $[2,3]$ while the second is to use an inverted pendulum to shift the centre of mass for static walking or the zero moment point for dynamic walking of the biped to a point within the area of the supporting foot $[5,6]$.

This paper presents a new strategy for dynamic walking where a gyroscope mounted on top of the biped is precessed to achieve balancing both in the Coronal and Sagittal planes. In this paper, only the kinematics and the stability of the gait of the biped will be considered. We will assume that the angles of the links of the biped can follow any prescribed reference trajectories precisely, and hence the dynamics of motion of the biped will not be considered.

\section{Biped Configuration}

Figure 1 illustrates the configuration of the biped under consideration in a 3D view (not to scale). The biped can be divided into two parts - the gyroscope and the legs. For clarity, the two d.c. motors driving the gyroscope, one for the rotation of the flywheel and the other for the precession of the flywheel axis, are not shown.

The directions of $x y z$ axes are defined as $x$-axis pointing to the right, $y$-axis to the front and $z$-axis to the top of the biped. The Coronal (frontal) plane is the $x z$ plane viewed from the front, the Sagittal (median) plane is the $y z$-plane viewed from the right and the Transverse (horizontal) plane is the $x y$-plane viewed from the top.

For a clear description of the current state of the biped, a fixed numbering scheme is applied - the links and the corresponding angles with the vertical are numbered from the left foot to the right foot as shown in Figure 2.

\section{Principle of Gyroscopic Couple}

In Figure 1, the wheel, of moment of inertia $J$, rotates about its axis $P U$ at $\omega \mathrm{rad} / \mathrm{s}$ and the axis is rotated (or precessed) in the horizontal plane $U P V$ about the axis $P W$ at $\Omega \mathrm{rad} / \mathrm{s}$. The angular momentum of the wheel, $J \omega$, about the axis of spin may be represented, in the usual convention, by the vector $P a$, the sense of the vector corresponding to the forward movement of a corkscrew turned in the direction of rotation of the wheel.

If the wheel is rotated about $P W$ through an angle $d \theta$, the angular momentum of the wheel is then 
represented by the vector $P b$ and the change in angular momentum by the vector $a b$, with a magnitude given by

$$
\text { change in angular momentum }=J \omega d \theta
$$

If this change takes place in time $d t$,

$$
\text { rate of change of angular momentum }=J \omega \frac{d \theta}{d t}
$$

This is called the gyroscopic couple and is denoted,

$$
C=J \omega \Omega
$$

Applying the corkscrew rule to the vector $a b$, the couple required to cause precession is directed along the $P V$ axis, counter-clockwise looking in the direction $V P$. The reaction to this couple, i.e. the couple exerted by the shaft $P U$ on its bearings, is opposite in direction to the applied couple.

Note that the vector representing the gyroscopic couple is perpendicular to the momentum vector of the wheel, with a sense opposing the precession $\Omega$. As an example, take the vector of the gyroscopic couple when the precession angle $\theta=-45^{\circ}$ and $\Omega>0$. Let the vector of the gyroscopic couple have magnitude $C$ and angle $\alpha$ measured from the $x$-axis. Figure 3 shows the top view of this situation. The vector representing the gyroscopic couple will be denoted as $C \angle \alpha$.

\section{Statement of the Problem}

Let $m_{i}$ be the mass of the $i$-th link and $\left(x_{i}, y_{i}, z_{i}\right)$ be the position of the centre of mass of the $i$-th link. With a gyroscopic couple $C \angle \alpha$ acting on the biped, the zero moment point (ZMP) on the plane $z=0$ is given by:

$$
\begin{aligned}
& x_{Z M P}=\frac{\left\{-\sum m_{i}\left(\ddot{z}_{i}+g\right) x_{i}+\sum m_{i} \ddot{x}_{i} z_{i}+C \sin \alpha\right\}}{\left\{\sum m_{i}\left(\ddot{z}_{i}+g\right)\right\}} \\
& y_{Z M P}=\frac{\left\{\sum m_{i}\left(\ddot{z}_{i}+g\right) y_{i}-\sum m_{i} \ddot{y}_{i} z_{i}+C \cos \alpha\right\}}{\left\{\sum m_{i}\left(\ddot{z}_{i}+g\right)\right\}}
\end{aligned}
$$

where $\ddot{x}_{i}, \ddot{y}_{i}$ and $\ddot{z}_{i}$ are, respectively, the second derivatives of $x_{i}, y_{i}$ and $z_{i}$, and $g$ is the gravitational constant.

Our objective is to find a gait for the biped, together with some associated motion for the gyroscopic couple so that the zero moment point (ZMP) is always within the foot of the supporting leg of the biped during the single support phase. The following constraints must be satisfied. a) The $y$-coordinate of the foot of the swinging leg should be larger at the end of the half walking cycle;

b) the $z$-coordinate of the foot of the swinging leg should not be less than zero during the cycle;

c) the angle of precession is continuous and monotonic increasing, and

d) the angular velocity of the wheel should have acceptable bounds.

\section{Balancing by Gyroscopic Motion}

Figure 4 shows the positions of the legs in a typical walking cycle, labelled from (1) to (10) in time order. The biped, at the time (1), has its left leg in front and right leg at the back with two legs standing. Through the time (2) to (4), it brings its right leg to the front. During the time (5) and (6), the biped stands with two legs, the right one in front and the left one at the back. During the time (7) to (9), the right leg becomes the supporting leg and the left leg is brought to front. Finally, the legs of the biped return to its initial states at the time (10). The biped carries out this sequence repeatedly.

In the time intervals (5) to (6), and (10) to (1), the biped stands with two legs, which are called the double support phase; otherwise, the biped stands with one leg, which is called the single support phase. Considered in the Coronal plane, when the biped stands on one leg, a moment should be supplied such that the zero moment point shifts to the supporting leg. During the single support phase, the lifting leg moving to front will produce moments in the Sagittal plane that has to be stabilized by a component of the gyroscopic couple.

The basic idea of our design is to generate the required couples from the gyroscope in order to:

a) create a sufficient moment in the Coronal plane to shift the ZMP under the sole of the supporting leg, so that the other leg can be lifted up, and

b) counteract the moment produced by the legs moving forward and backward in the Sagittal plane.

Table 1 shows how the motion of the gyroscope should be synchronized with the movement of the legs as given in Figure 4 in order to balance the biped in both the Coronal and Sagittal planes.

Initially, at time (1), the precession angle is at $\theta=-90^{\circ}$, which means that the vector of the angular momentum of the rotating wheel is pointing to the back of the biped, and the precession of the gyroscope is in a counter-clockwise direction. The precession angle is 
increased continuously through $\theta=0^{\circ}$ at time (3) to $\theta=90^{\circ}$ at time (5) to complete half a walking cycle. When the momentum vector reaches the angle $\theta=90^{\circ}$ (pointing to the front of the biped), the precession of the gyroscope is reversed and it moves back in the clockwise direction. Finally, it returns back to $\theta=-90^{\circ}$ at time (10) to complete a cycle of walking.

Note that the entries in Table 1 are labelled (1) - (10) in accordance with the leg positions given in Figure 4. The arrows beside the time labels indicate the direction of the gyroscopic couple in the Transverse plane. For example the direction of the couple at time (2) is as illustrated in Figure 3. It can be shown that the moment produced by the biped at time (2) is generally in a direction opposite to the gyroscopic couple. If this couple is controlled appropriately, an exact cancellation of the moment of the legs and the gyroscope couple can occur. Therefore, the biped can walk in a dynamically stable manner.

\section{Verification by Simulation}

The above discussion provides a qualitative basis for solving the balancing problem by means of a gyroscope. We still need to produce a gait that will satisfy all the constraints stated in section 4 .

We have designed a number of gaits and checked each gait with the above constraints to determine whether it is feasible or not. After a large number of trials, we found that the constraints are quite restrictive. Figures 59 show one possible gait obtained by a heuristic argument. In Figures 5-9, $\theta_{i}(i=1, \ldots, 5)$ the trajectories of the angles of the links in a half walking cycle are given (with a half period of 1.05 seconds). This half walking cycle corresponds to the sequence marked as (1) to (5) in the Figure 4. Table 2 gives the parameters (masses and lengths of the links) of the biped. Based on the information of the angles and the parameters, the centre of mass $\left(x_{i}, y_{i}, z_{i}\right)$ for the links and the position the foot of the swinging leg are obtained by kinematic equations and the results are plotted in Figures 10-12. The two components ( $C \sin \alpha$ and $C \cos \alpha$ ) of the required gyroscopic couple can be deduced by equating the ZMP (see section 4) to the coordinates of the supporting foot. Then, the angle of precession and its rate (Figures 13-14) and the angular velocity of the wheel (Figure 15) are determined.

From Figures 10-15, it can be seen that all the constraints are satisfied. Therefore, Figures 5-9 represent a feasible gait where a gyroscopic couple can be used to balance the biped in dynamic walking.

\section{Further Work}

Although we have shown by construction a gait for stable dynamic walking, there is scope for improvement. In our simulation, the supporting sole is taken to be a point. As a result, the position of the zero moment point is restricted to coincide with the point sole of the supporting leg. This implies that both the magnitude and direction of the gyroscopic couple is uniquely determined. This leads to some unsatisfactory features in the solution given in Figures 5-15. For example,

(a) both $\omega$ and $\Omega$ have jump discontinuities (see Figures 14 and 15), and

(b) the trajectory of the swinging leg is unnatural and is too close to the ground (see Figure 12).

An obvious modification for improving the solution is to replace the point sole by a rectangular sole. The position of the zero moment point can then be relaxed to lie within the area of the sole. How the degree of freedom in placing the position of the zero moment point can be used to improve the posture of walking or to simplify the operation of the gyroscope is a topic for further investigation.

\section{Conclusion}

We have presented a new strategy for biped dynamic walking using a gyroscopic couple for balancing. Its feasibility has been verified by simulation. An advantage of the proposed method is that unlike the conventional method of trunk motion, our method simplifies the model of the biped from a complex kinematic chain to a simple one. This is because the use of a gyroscope for balancing eliminates the need to have branching links at the hip of the biped as would be required in a balancing scheme using an inverted pendulum.

\section{References}

[1] T. Emura and A. Arakawa, "A Study on Walking Robots Controlled with Attitude Sensors", Advanced Robotics, 1989, pp 640-651

[2] J. Furusho and M. Masubuchi, "Control of a Dynamical Biped Locomotion System for Steady Walking", $J$. of Dyn. Sys., Man, and Control, 1986, Vol. 108, pp 111-118

[3] T. Mita and et al, "Realization of a High Speed Biped Using Modern Control Theory", Int. J. of Control, 1984, Vol. 40, No.1, pp 107-119

[4] P. P. Schilovsky, The Gyroscope: its Practical Construction and Application, 1938, E. \& F. N. SPON 
[5] A. Takanishi and et al, "The Realization of Dynamic Walking by the Biped Walking Robot WL-10RD", ICAR, 1985, pp 459-466

[6] A. Takanishi and et al, "Realization of Dynamic Biped Walking Stabilized with Trunk Motion Under Known External Force", Advanced Robotics, 1989, pp 299-310

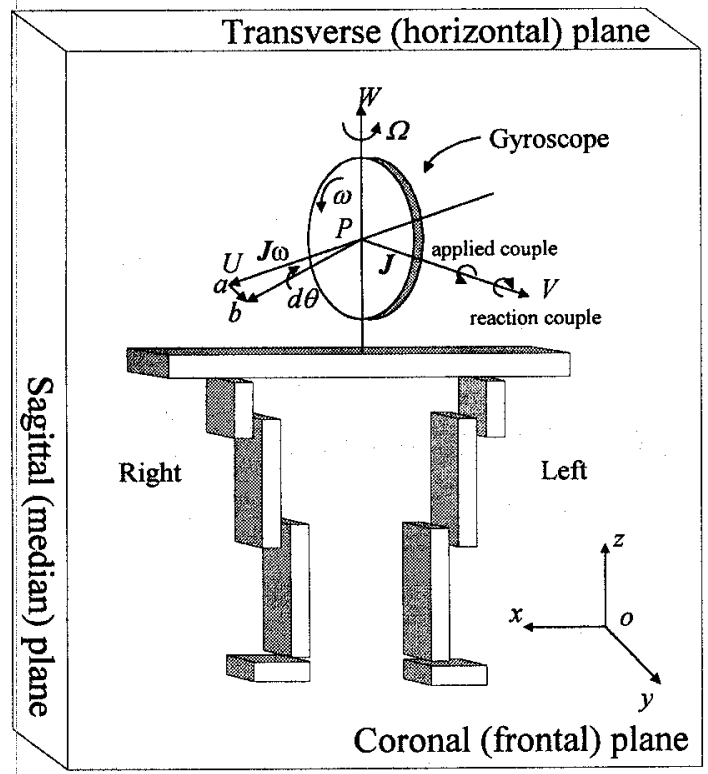

Figure 1 - 3D View of the Biped

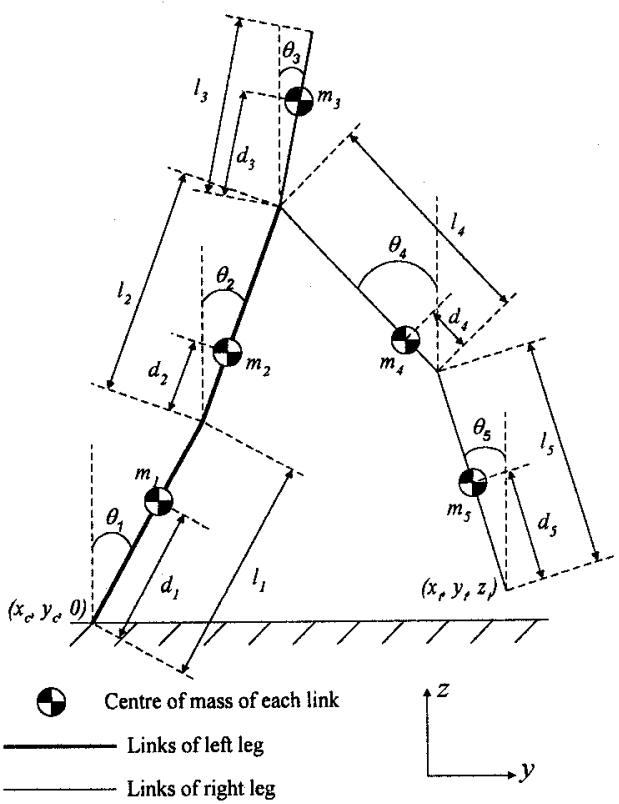

Figure 2 - Model of the Biped

\begin{tabular}{|c|c|c|}
\hline $\begin{array}{c}\text { Precession } \\
\text { Angle } \theta \\
\text { from }-90^{\circ} \text { to } 90^{\circ}\end{array}$ & $\begin{array}{c}\text { Counter- } \\
\text { clockwise } \\
\text { Precession } \\
\text { i.e., Increasing } \theta \\
\text { Positive } \Omega\end{array}$ & $\begin{array}{c}\text { Clockwise } \\
\text { Precession } \\
\text { i.e., } \\
\text { Decreasing } \theta \\
\text { Negative } \Omega \\
\end{array}$ \\
\hline $90^{\circ}$ & $(5) \leftarrow$ & $(6) \rightarrow$ \\
\hline $45^{\circ}$ & (4)K & (7)y \\
\hline $0^{\circ}$ & (3) $\uparrow$ & $(8) \downarrow$ \\
\hline$-45^{\circ}$ & (2) $\pi$ & (9)K \\
\hline$-90^{\circ}$ & $(1) \rightarrow$ & $(10) \leftarrow$ \\
\hline
\end{tabular}

Table 1 - Directions of Vectors of Gyroscopic Couple Generated

\begin{tabular}{|l|l|l|}
\hline & Links & Values \\
\hline \multirow{5}{*}{ Lengths } & $l_{1}$ & $0.15 \mathrm{~m}$ \\
\cline { 2 - 3 } & $l_{2}$ & $0.15 \mathrm{~m}$ \\
\cline { 2 - 3 } & $l_{3}$ & $0.10 \mathrm{~m}$ \\
\cline { 2 - 3 } & $l_{4}$ & $0.15 \mathrm{~m}$ \\
\cline { 2 - 3 } & $l_{5}$ & $0.15 \mathrm{~m}$ \\
\cline { 2 - 3 } & $\begin{array}{l}l_{w} \text { (between two } \\
\text { legs) }\end{array}$ & $0.05 \mathrm{~m}$ \\
\cline { 2 - 3 } & $d_{1}$ & $0.075 \mathrm{~m}$ \\
\hline$d_{2}$ & $0.02 \mathrm{~m}$ \\
\hline$d_{3}$ & $0.05 \mathrm{~m}$ \\
\hline$d_{4}$ & $0.02 \mathrm{~m}$ \\
\hline$d_{5}$ & $0.075 \mathrm{~m}$ \\
\hline \multirow{5}{*}{ Masses } & $m_{1}$ & $0.4 \mathrm{~kg}$ \\
\hline$m_{2}$ & $0.4 \mathrm{~kg}$ \\
\cline { 2 - 3 } & $m_{3}$ & $0.9 \mathrm{~kg}$ \\
\cline { 2 - 3 } & $m_{4}$ & $0.4 \mathrm{~kg}$ \\
\cline { 2 - 3 } & $m_{5}$ & $0.4 \mathrm{~kg}$ \\
\hline
\end{tabular}

Moment of inertia of the rotating wheel of mass $0.5 \mathrm{~kg}$ and radius $0.05 \mathrm{~m}, J=0.000625 \mathrm{kgm}^{2}$

The lateral inclination., $\phi=0$,

The contact point of the supporting leg is: $x_{C}=0, y_{C}=0$, and $z_{C}=0$.

Duration of Single-Support Phase $=1.05 \mathrm{sec}$.

Table 2 - The Parameters of the Biped 


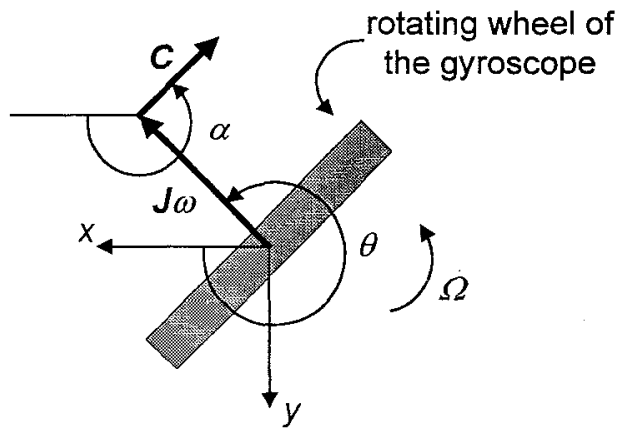

Figure 3 - Top View of the Gyroscope
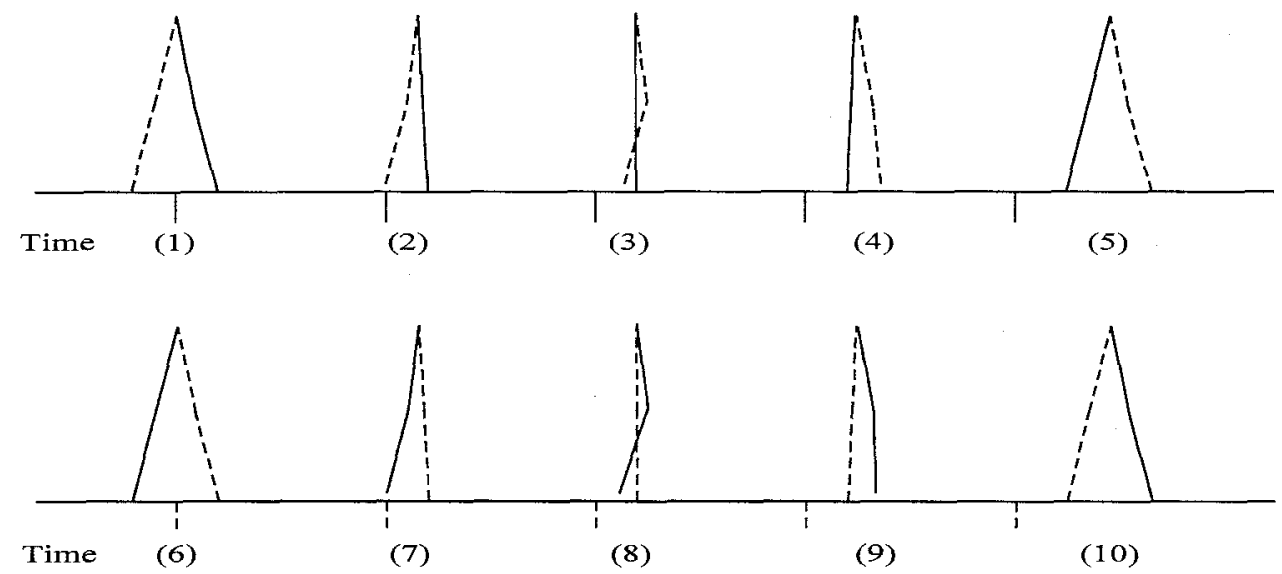

Legend: —— Left leg _-_-_--. Right leg

Figure 4 - Positions of Legs during a Walking Cycle

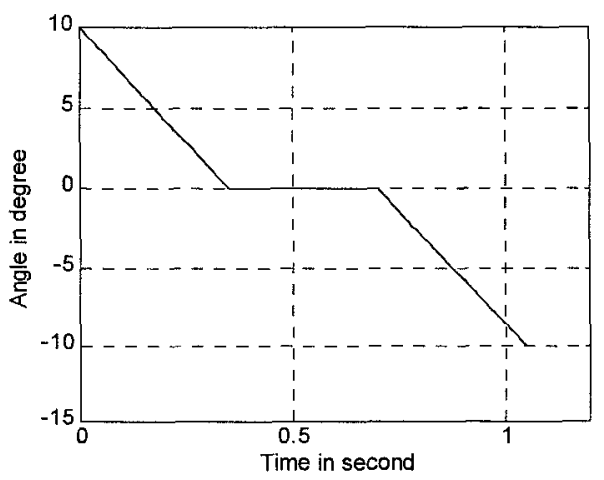

Figure 5 - The Trajectory of $\theta_{1}$

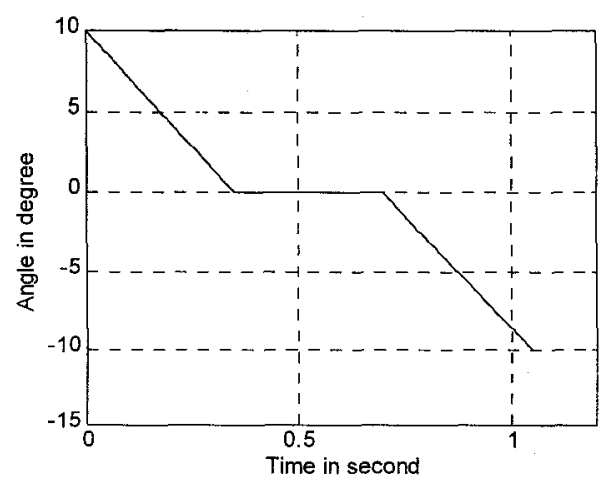

Figure 6 - The Trajectory of $\theta_{2}$ 


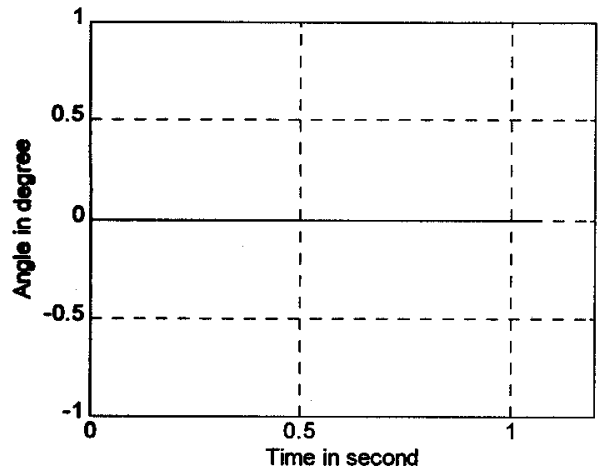

Figure 7 - The Trajectory of $\theta_{3}$

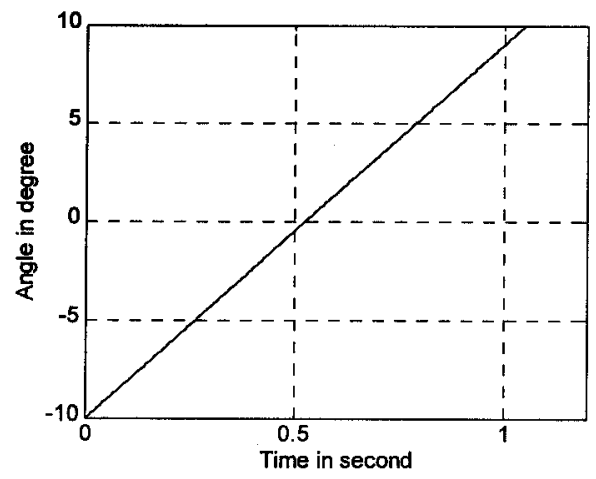

Figure 8 - The Trajectory of $\theta_{4}$

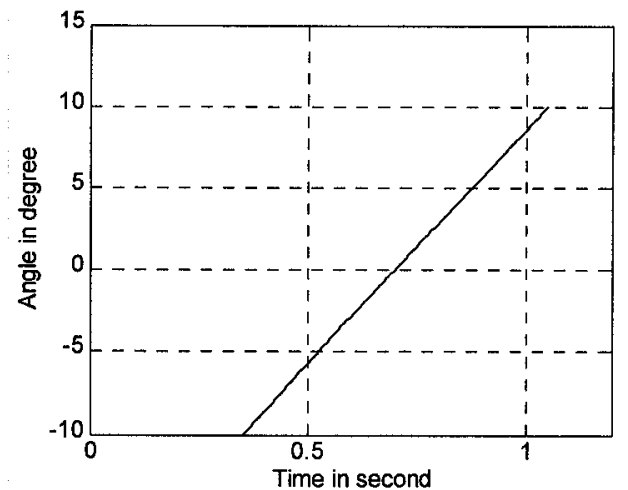

Figure 9 - The Trajectory of $\theta_{5}$

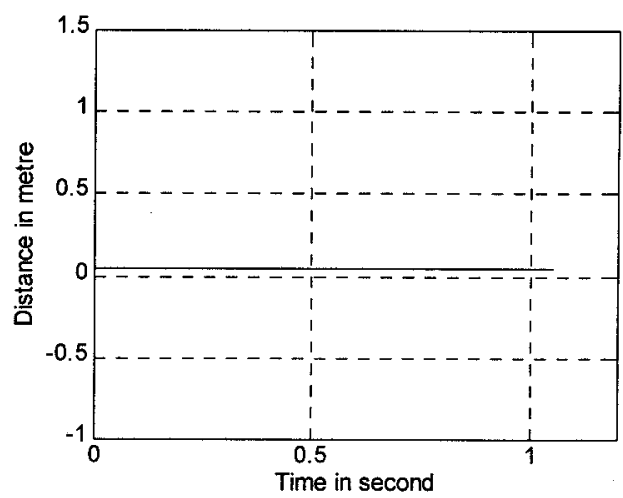

Figure 10 - The $x$-coordinate of the Foot of the Swinging Leg

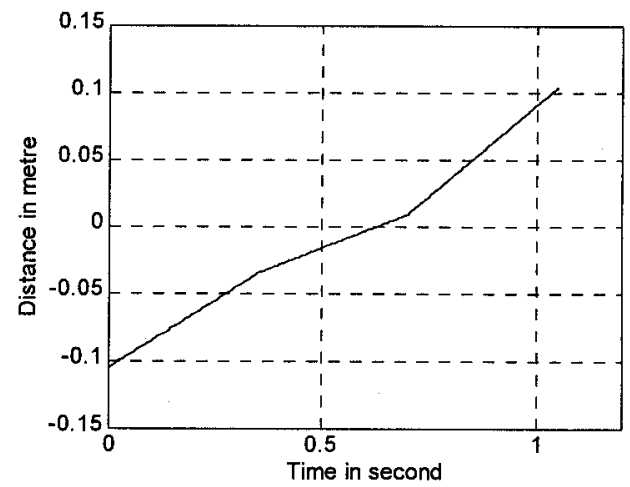

Figure 11 - The $y$-coordinate of the Foot of the Swinging Leg

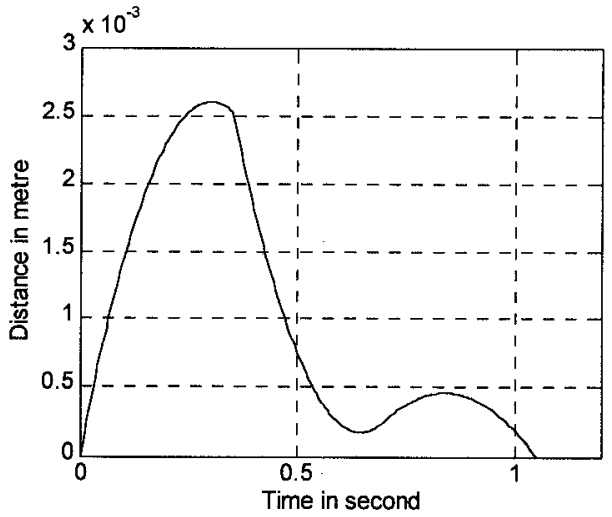

Figure 12 - The z-coordinate of the Foot of the Swinging Leg 


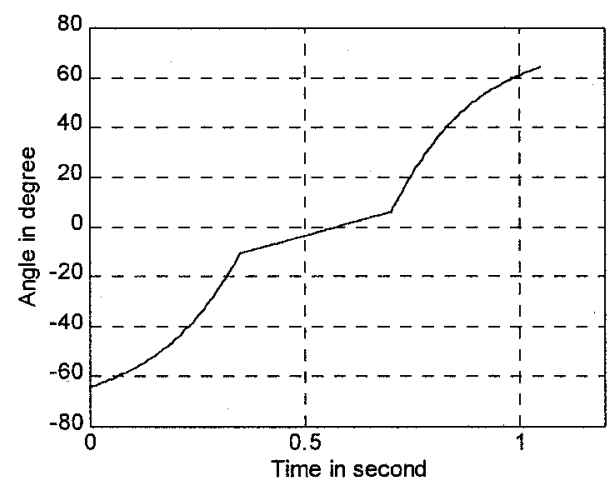

Figure 13 - The Angle of Precession of the Gyroscope, $\theta$

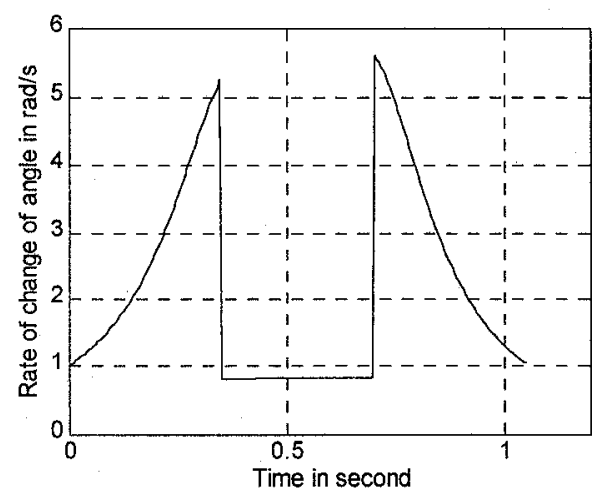

Figure 14 - The Rate of Change of the Precession Angle, $\Omega$

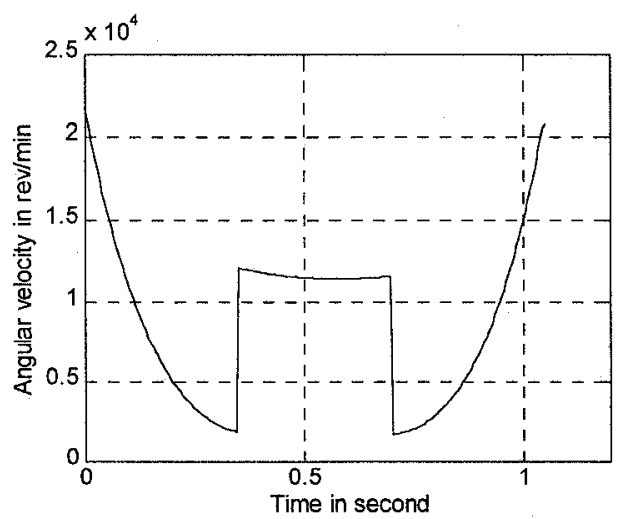

Figure 15 - The Angular Velocity of the Wheel of the Gyroscope, $\omega$ 